Science@Direct

Consciousness and Cognition 15 (2006) 323-341
Consciousness and Cognition

www.elsevier.com/locate/concog

\title{
Mere exposure effect: A consequence of direct and indirect fluency-preference links is
}

\author{
Sylvie Willems ${ }^{\mathrm{a}, *}$, Martial Van der Linden ${ }^{\mathrm{a}, \mathrm{b}}$ \\ a University of Liège, Belgium \\ ${ }^{\mathrm{b}}$ University of Geneva, Switzerland
}

Received 10 February 2005

Available online 22 September 2005

\begin{abstract}
In three experiments, picture quality between test items was manipulated to examine whether subjects' expectations about the fluency normally associated with these different stimuli might influence the effects of fluency on preference or familiarity-based recognition responses. The results showed that fluency due to pre-exposure influenced responses less when objects were presented with high picture quality, suggesting that attributions of fluency to preference and familiarity are adjusted according to expectations about the different test pictures. However, this expectations influence depended on subjects' awareness of these different quality levels. Indeed, imperceptible differences seemed not to induce expectations about the test item fluency. In this context, fluency due to both picture quality and pre-exposure influenced direct responses. Conversely, obvious, and noticed, differences in test picture quality did no affect responses, suggesting that expectations moderated attributions of fluency only when fluency normally associated with these different stimuli was perceptible but difficult to assess.
\end{abstract}

(c) 2005 Elsevier Inc. All rights reserved.

Keywords: Fluency; Mere exposure effect; Familiarity; Picture quality; Expectations

\footnotetext{
S. Willems is a Research Fellow with the Belgian National Fund for Scientific Research (FNRS).

* Corresponding author. Fax: +32 43662875 .

E-mail address: sylvie.willems@ulg.ac.be (S. Willems).
} 


\section{Introduction}

Repeated exposure to an initially neutral and unfamiliar stimulus, without any reinforcement, results in more favorable affective judgment, a phenomenon known as the mere exposure effect (Zajonc, 1968). Experiments involving a wide range of stimuli (e.g., Chinese ideographs, Turkish words, line drawings, faces, possible and impossible three-dimensional objects, and melodies), and procedures (from forced-choice preference judgments to likeability ratings, pleasantness ratings, behavioral indices of stimulus preference, and self-reports of mood) have shown that people frequently prefer stimuli they have seen before over novel stimuli (see Bornstein, 1989; Harrison, 1977, for reviews).

Several researchers suggested that enhanced processing fluency due to a previous encounter may serve as a basis for preference and produce the mere exposure effect (e.g., Bornstein \& D'Agostino, 1994; Jacoby, Kelley, \& Dywan, 1989; Seamon, Brody, \& Kauff, 1983a, Seamon, Brody, \& Kauff, 1983b; Whittlesea, 1993; Whittlesea \& Price, 2001). Enhanced processing fluency can be defined as the ease with which information can be processed (as reflected by the speed and ease with which a stimulus is perceived), either at a perceptual level (i.e., subsequent to a perceptual encoding process involving that stimulus: perceptual fluency) or at a conceptual level (i.e., subsequent to a conceptual process involving information related to that stimulus: conceptual fluency). If processing fluency due to a prior encounter is central to the mere exposure effect, Schwartz, Reber, Winkielman and collaborators (e.g., Reber \& Schwarz, 2001; Reber, Schwarz, \& Winkielman, 2004; Schwarz, 2004a; Winkielman, Schwarz, Fazendeiro, \& Reber, 2003) assume that any other variables that facilitate fluent processing should similarly increase preference, even without pre-exposure. In agreement with this assumption, several studies have shown that a host of variables - such as figure-ground contrast, clarity with which a stimulus is presented, symmetry, presentation duration, presentation of a subliminal prime just before the stimulus, etc.-influence the ease of processes as well as preference judgments (e.g., Reber et al., 2004, for a review). Finally, recent research has shown that repeated pre-exposure, as well as other fluency manipulations (e.g., presentation of a subliminal prime just before the stimulus, variations in presentation duration), does not affect only explicit preference judgments but also elicits psychophysiological responses (Harmon Jones \& Allen, 2001; Winkielman \& Cacioppo, 2001) like positive affective responses over the region of the zygomaticus major ("smiling muscle") as measured by facial electromyography (EMG; e.g., Cacioppo, Petty, Losch, \& Kim, 1986). Consequently, in the case of mere exposure effect paradigms, preference most probably results from processing fluency due to repeated preexposure. However, the latter is not central per se for preference judgment. Rather, any variable that increases processing fluency could elicit spontaneous affective reactions, which, in turn, could serve as relevant input for perceivers' explicit preference judgment (e.g., Reber et al., 2004; Schwarz, 2004a; Winkielman et al., 2003).

Furthermore, although this link between fluency and preference has been well established, and even though the mere exposure effect has proven to be a reliable phenomenon, the influence of fluency on explicit preference judgment is not always observed and seems to depend on several experimental conditions (e.g., see Bornstein, 1989, for some boundary conditions). For example, it has been observed that pre-exposure has the strongest influence on explicit preference when stimuli are presented during encoding for relatively short durations (e.g., Bornstein \& D'Agostino, 1992), with low pre-exposure frequency (e.g., Van den Bergh \& Vrana, 1998), or when the stimuli 
used are complex (Bornstein, Kale, \& Cornell, 1990; Cox \& Cox, 1988, 2002). It also appears that people sometimes prefer novel to familiar stimuli (Fenske, Raymond, \& Kunar, 2004), complex rather than simple figures (Berlyne \& Crozier, 1971), and so on. These observations may reflect the intermediation of additional indirect factors such as attribution, expectation, and relevance evaluation between automatic positive affect due to processing fluency and explicit preference response (see Reber et al., 2004; Winkielman et al., 2003).

The literature on fluency as a cue to familiarity-based recognition may shed some light on these additional indirect factors. According to the fluency/attribution account (e.g., Kelley \& Jacoby, 1998), familiarity is explained in terms of unconscious attribution of stimulus fluency to the effect of a prior experience; the stimulus is then consciously experienced as familiar. However, fluency as a cue to familiarity does not operate as a simple equation "fluent stimulus equals old stimulus" (e.g., Whittlesea, 2003). On the contrary, the relationship between the feeling of familiarity and fluency seems to be indirect, mediated by unconscious attributional processes (Jacoby \& Dallas, 1981; Jacoby et al., 1989). For example, Kelley and Rhodes (2002) suggest that when an irrelevant source of fluency (e.g., the presentation of an obvious supraliminal prime just before the stimulus) is noticed and appreciated by the subject, high-processing fluency may be perceived as uninformative and may be entirely attributed to this irrelevant source (i.e., to the prime at the test time and not to a previous encounter); accordingly, it will not give rise to a feeling of familiarity. Further, a recent series of works by Whittlesea and colleagues (Whittlesea \& Leboe, 2003; Whittlesea \& Williams, 1998, 2000, 2001a, 2001b; see also Lloyd, Westerman, \& Miller, 2003; Westerman, Lloyd, \& Miller, 2002) provide evidence that these attributional processes are quite sophisticated. According to their discrepancy-attribution hypothesis, enhanced fluency is attributed to prior exposure to a stimulus, and creates a feeling of familiarity, only when the individual is surprised by the ease with which he or she is able to process the item. In other words, their hypothesis states that an individual interprets fluency as a sign of previous encounter if the actual fluency he or she perceives for the stimulus is greater than the fluency that would normally be expected from that type of stimulus. Thus, seeing a student in class does not generate a striking feeling of familiarity, whereas seeing the same student at the supermarket does.

In the same vein, some authors have suggested that, once affective experience is elicited by a fluent processing, its impact on preference judgments may be moderated by indirect attributional processes (e.g., Bornstein \& D'Agostino, 1994; Butler, Berry, \& Helman, 2004; Reber et al., 2004; Schwarz, 2004a; Van den Bergh \& Vrana, 1998; Winkielman \& Fazendeiro, 2003, cited by Reber et al., 2004). Butler et al. (2004) recently obtained a mere exposure effect for nonwords but not for words. These authors proposed that, in the case of real words, participants may expect to be able to process such stimuli efficiently. As such, the enhanced fluency associated with recently presented words is unsurprising and is not attributed to preference. Conversely, enhanced fluency for nonwords may be more surprising and capture subjects' attention; this increases the likelihood that fluency will serve as the basis for preference decisions. However, another possibility suggested by these authors is that preferences for words may already be fully established before the start of the experiment and therefore it is unlikely to have been changed by a single experimental exposure. Further, Winkielman and Fazendeiro gathered more direct evidence for attributional processes' contribution by demonstrating that the influence of fluency on liking judgments is eliminated when subjects attribute their first affective reaction to an external and irrelevant variable, such as background music. 
Nonetheless, earlier findings showed that, even when a feeling of fluency might be expected, some fluently processed stimuli may continue to elicit an explicit preference, such as after an obvious supraliminal presentation phase, of the kind that has been used in some mere exposure paradigms (e.g., Seamon et al., 1995, 1997). Consequently, the nature of the connection between ease of processing and explicit preference judgments and the importance of additional indirect factors such as expectations and attributions of perceived fluency seem to be complex. The role of such indirect factors in the fluency-preference link has not received much attention (see Winkielman et al., 2003); nevertheless, their investigation could help us to understand the mere exposure effect.

The first question addressed by this study was whether the impact on explicit preference judgments of enhanced fluency due to pre-exposure (i.e., the mere exposure effect) may be modulated by subjects' expectations about the fluency feeling during their judgment (Butler et al., 2004; Reber et al., 2004). For example, subjects' expectations could be manipulated by different levels of picture quality during the preference judgment. Indeed, people most probably expect greater fluency for pictures that are presented with a high level of picture quality than for lower-quality pictures. We assumed that subjects' expectations due to picture quality could have two potential influences (Schwarz, 2004a, 2004b). First, an unexpected fluency feeling (e.g., fluency due to pre-exposure for a low-quality stimulus) might be more salient and might be more likely to be used for preference judgments. Second, if a variable (e.g., a stimulus presented with a high picture quality) allows participants to formulate expectations about the feeling of fluency, this feeling might be attributed mainly to this variable. In this case, there are two options: either this variable is perceived as relevant (e.g., an objective characteristic of the object itself, such as its symmetry), and the positive affect related to perceived fluency is considered as an useful cue to preference judgment. Alternatively, this variable might be perceived as incidental and irrelevant (e.g., picture quality, or any other variable that is not inherent in the object itself), and thus the resulting positive affect related to fluency is evaluated as uninformative and subjects might turn to inputs other than fluency to form a preference judgment (e.g., Bornstein \& D'Agostino, 1994; Schwarz, 2004a, 2004b; Van den Bergh \& Vrana, 1998).

The second question addressed was whether the potential influence of subjects' expectations on the mere exposure effect depended on subjects' awareness of the different levels of picture quality. We assumed that the fluency feeling that derives from picture quality might have three potential impacts. First, an imperceptible variation in quality should simply have a direct and independent impact on the preference judgment. Indeed, with an imperceptible difference in quality, participants will probably not construct expectations concerning the ease of processing high- and low-quality stimuli. Consequently, the absolute amount of processing fluency (i.e., due to both pre-exposure and picture quality effects) should influence their judgment. Similar results for familiarity have often been observed by using an imperceptible factor to enhance fluency for a portion of the test items (e.g., Jacoby \& Whitehouse, 1989; Lloyd et al., 2003; Westerman, 2001). Second, a more perceptible but still small quality difference might allow participants to expect to be able to process stimuli more or less efficiently. So, as described above, an unexpected feeling of fluency (such as enhanced fluency for recently presented low-quality stimuli) should elicit an explicit preference. Conversely, the influence of fluency feelings associated with high-quality stimuli should be reduced, because this fluency should be less surprising and thus should be attributed mainly to picture quality. Finally, a more obvious and noticeable quality difference could allow subjects to allocate more resources to accurately assess the fluency resulting from picture quality by 
comparing the relative fluency for a specific stimulus to other stimuli with the same picture quality. Thus, the mere exposure effect size should not be influenced by a too obvious difference in picture quality between stimuli, or at any rate, it should be less so.

To examine whether the influence of indirect factors could be generalized and applied to other feelings that depend on processing fluency, the third goal of this study was to compare the impact of these indirect factors on the mere exposure effect, on one hand, and on the feeling of familiarity in a recognition task, on the other hand. To explore these questions, we investigated the mere exposure effect and familiarity-based recognition by creating test conditions where fluency was modulated for target items during the test phase. To this end, we presented unfamiliar three-dimensional objects in the study phase with incidental encoding. Then, we investigated the influence of various picture qualities by manipulating the degree of figure-ground contrast for targets and/ or distractors during forced-choice preference and recognition judgments. In addition, a Remember-Know-Guess (RKG) procedure was applied to the recognition task.

The reasons for these methodological choices were as follows. First, Seamon et al. (1995, 1997) have shown a significant mere exposure effect by using the same kind of unfamiliar three-dimensional objects with supraliminal presentation and incidental encoding. In addition, it has been shown that pictures with a high figure-ground contrast are perceived as clearer and easier to process than low-contrast ones (Checkosky \& Whitlock, 1973; Whittlesea, Jacoby, \& Girard, 1990). Moreover, studies have shown that preference judgments about simple circles are influenced by fluency due to variation in figure-ground contrast (Reber \& Schwarz, 2001; Reber, Winkielman, \& Schwarz, 1998). However, this influence could differ depending on the kind of figure to be processed. Indeed, while picture quality may be judged pertinent by subjects for their preference judgments of relatively simple figures (such as circles; Reber \& Schwarz, 2001; Reber et al., 1998), it may be perceived as non-essential and incidental for complex three-dimensional objects. Indeed, this material is inherently rich in visual features that subjects should perceive as more essential to their preference judgments than picture quality. In addition, the extent of figure-ground contrast can easily be manipulated. Thus, we can compare the influence of contrast between targets and/or distractors with imperceptible $(10 \%$, Experiment 1), subtle (20\%, Experiment 2), or obvious (40\%, Experiment 3) manipulations. Fourth, the forced-choice procedure allows one to manipulate the subject's expectation of a fluency advantage or disadvantage for the target by direct comparison with the distractor. For example, with a distractor with a low-contrast level, the fluency advantage for the target might be more expected when the target is presented with a higher level of contrast than with the same low level. Conversely, a fluency advantage for the target might be more surprising if the target is presented with a lower contrast level than the distractor. Fifth, we used the Remember-Know-Guess recognition procedure (RKG; Gardiner, 1988; Tulving, 1985) in order to characterize the processes involved in recognition performance. The procedure requires subjects to report their states of awareness accompanying the act of retrieval. If they can recollect some discriminating details of the item itself or of the learning context, they make a Remember (R) response (recollection process). If the participants simply have a feeling of familiarity, in the absence of any detailed recollection, they make a Know (K) response. Finally, if they are unsure about their response, they make a Guess $(\mathrm{G})$ response. Past research has shown that processing fluency can mediate successful $\mathrm{K}$ responses, but not $\mathrm{R}$ recognition (e.g., Rajaram, 1993; Rajaram \& Geraci, 2000). 


\section{Experiment 1}

In this first experiment, we examined cases in which processing fluency is enhanced by pre-exposure and additionally manipulated by imperceptible figure-ground contrast variations at the time of preference and recognition judgments. We assumed that enhancing or decreasing fluency by imperceptible manipulations of the figure-ground contrast could have a direct impact on the mere exposure effect and on familiarity-based recognition. More specifically, for the test phase, we prepared four types of target-distractor pairs by combining low- or high-contrast distractors (Distractor-/+) and low- or high-contrast targets (Target-/+). In terms of absolute fluency advantage for targets, Distractor-/Target+ pairs should be superior to Distractor-/Targetand Distractor+/Target + pairs, which themselves should be superior to Distractor+/Targetpairs. Indeed, for Distractor-/Target + pairs, there is a facilitation of processing only for the target due to pre-exposure and high contrast at the time of testing. Conversely, for Distractor+/Target - pairs, there is a facilitation of target processing due to pre-exposure; however, the distractor is also made easier to process when it has a higher contrast than the target. Finally, for Distractor-/Target- and Distractor $+/$ Target + , target and distractor did not differ in contrast; only pre-exposure could enhance target fluency.

\subsection{Method}

\subsubsection{Subjects}

Fourteen undergraduate students were volunteers in this experiment. There were six men and eight women. Their mean age was 20.2 years $(S D=1.3)$.

\subsubsection{Materials and procedure}

Forty-eight line drawings of unfamiliar three-dimensional objects were used (Williams \& Tarr, 1997). In a preliminary phase, we prepared two sets of equally complex figures (see Carrasco \& Seamon, 1996). First, each figure was rated by 30 undergraduate students on a 5-point Likert scale of "subjective complexity." Then, angles, elements, lines, and segments were counted for each figure. Figures were assigned to Sets A and B so that they would be equal on all these variables $(p s>.05)$. Half of the subjects were presented with Set A as targets and Set B as distractors. The other half of the subjects were presented with the reverse design. Thus, the target and distractor sets did not differ with regard to the objective and subjective complexity measures.

We created two versions of each figure: high-contrast (white on black) and low-contrast (the picture underwent a 10\% contrast reduction). This contrast reduction was very slight; thus, subjects should not perceive the decrease in contrast quality. High-contrast versions were used for study lists. For the test phase, we prepared 24 equally complex pairs of target-distractor figures: 6 Target $+/$ Distractor-, 6 Target-/Distractor-, 6 Target $+/$ Distractor + , and 6 Target-/Distractor + pairs. Stimuli that were assigned to these four contrast conditions were randomly counterbalanced between subjects.

To verify the extent to which the $10 \%$ contrast reduction is or is not perceptible by subjects, we obtained judgments of contrast perception online from 10 additional subjects. More specifically, these 10 subjects were asked to rate the pairs that were used in Experiment 1 on a 4-point Likert scale that assessed conscious "contrast difference" perception (1: I don't see contrast difference 
between the two figures; 2 : I have a vague feeling of contrast difference between the two figures; 3 : I see a slight difference between the two figures; 4 . I see a very clear difference between the two figures). In addition, subjects were asked to choose the most contrasted stimulus within each pair; even if they did not see any difference between the two stimuli.

The results showed that there is practically no conscious "contrast difference" perception. More precisely, for pairs composed of distinct contrast stimuli, of the 120 responses, 88 responses were of type "1" ("I don't see contrast difference"); 22 responses "2" ("I have a vague feeling of contrast difference"), and 10 responses " 3 " ("I see a slight difference"). The mean of contrast perception on the 4-point Likert was thus only $1.35(S D=.57)$. More importantly, identical results were obtained for the pairs composed of stimuli with same contrast. Indeed, the mean of contrast perception estimation on the 4-point Likert for these pairs was $1.3(S D=.56)$. Thus, the "contrast difference" perception estimation did not differ between pairs composed of same contrast stimuli and pairs composed of distinct contrast stimuli $(t(9)<1)$. Second, for the pairs composed of distinct contrast stimuli, subjects correctly chose the most contrasted stimulus in $54 \%$ of the trials, which was only marginally above chance $(t(9)=1.581, p<.1, d=.50)$. However, these few correct responses did not differ from incorrect responses as regards "contrast difference" perception estimation (respectively, correct responses $-M=1.43, S D=.44$; incorrect responses $-M=1.48, S D=.68 ; t(9)<1)$.

The material was presented using E-Prime software (Psychology Software Tools, Inc.) on a 17 in. color monitor running at $60 \mathrm{~Hz}$, approximately $70 \mathrm{~cm}$ from the subject. Figures were about $6 \mathrm{~cm}$ in height and $6 \mathrm{~cm}$ in width.

\subsubsection{Incident study phase}

Participants were told that the study involved "object perception," and that they were going to see 72 figures. After viewing three examples, they were asked to estimate the orientation for each figure appearing on the screen (left or right). This task ensures that the subject stays focused throughout the presentation and that global perceptual encoding takes place (inducing structural processing of three-dimensional objects; Schacter, Cooper, \& Delaney, 1990). No mention was made of any subsequent memory test.

Subjects were then shown 24 white-on-black figures, three times each, in three random orders of 24. Each study stimulus was presented at the center of the screen for $2500 \mathrm{~ms}$, followed by a $3500 \mathrm{~ms}$ interval (see Seamon et al., 1995, 1997, for a similar procedure).

\subsubsection{Forced-choice preference judgment}

Subjects were randomly presented with the 24 target-distractor pairs. Both figures were presented simultaneously to the subject for $1500 \mathrm{~ms}$ followed by a self-spaced interstimulus interval. This relatively short duration was used to encourage reliance on fluency of processing (e.g., Reber \& Schwarz, 2001). The side of the screen in which the target stimulus was displayed was randomized over trials. Subjects were asked to examine each pair and to choose the geometric shape they liked best.

\subsubsection{Forced-choice recognition judgment}

The same pairs were displayed as in the preference task. Subjects were asked to point to the shape that they had seen during the exposure phase. The procedure was identical to the preference 
judgment except that the subjects were asked to make RKG response. Written instructions explaining RKG responses were given to participants (see Appendix A). Participants entered their recognition responses on the computer keyboard and made the RKG judgments orally.

Half of the subjects were presented with the preference judgment in first position and the recognition judgment afterwards. The other half of the subjects were presented with the reverse design. The participants were tested individually.

\subsection{Results and discussion}

The mean proportion of target objects selected in the recognition and preference tasks as a function of the four contrast conditions is presented in Table 1. As there was no effect of the order of the tasks on preference and recognition scores $(p s>.1)$, we did not consider this variable in the following analyses. This might appear surprising. Indeed, the first test provides exposure to the distractors, which should increase their fluency. However, the targets were also presented during the first test. So, even if the first test had increased distractors' fluency, it should also have increased targets' fluency, thus leaving the fluency difference between targets and distractors unchanged between the first and the second test.

\subsubsection{Mere exposure effect}

We observed a significant mere exposure effect (subjects selected the previously seen figures in a proportion greater than chance, estimated at .50), with medium-sized effects for Target-/Distractor- $(t(13)=1.873$, one-tailed, $p<.05, d=.50)$, and for Target $+/$ Distractor $+(t(13)=1.803$, $p<.05, d=.48)$, and a large effect for Target $+/$ Distractor $-(t(13)=3.426, p<.005, d=.92)$. The three conditions showing a significant mere exposure effect were not significantly different $(p>.1)$. However, as expected, we observed a particularly large preference effect size for pairs with a facilitation of processing only for targets due to both pre-exposure and high contrast at test (Target+/Distractor - pairs). Indeed, Cohen (1988) defined effect sizes as "small, $d=.2$," "medium, $d=.5$," and "large, $d=.8$." Moreover, we observed no significant mere exposure effect for

Table 1

Means as a function of the four contrast conditions for Experiments 1, 2, and 3

\begin{tabular}{|c|c|c|c|c|}
\hline \multirow[t]{2}{*}{ Tasks } & \multicolumn{2}{|c|}{ Distractors - } & \multicolumn{2}{|c|}{ Distractors +} \\
\hline & Targets - & Targets + & Targets - & Targets + \\
\hline \multicolumn{5}{|c|}{ Experiment 1: $10 \%$ of contrast difference } \\
\hline Preference & $.61(.23)$ & $.70(.22)$ & $.49(.28)$ & $.60(.21)$ \\
\hline Recognition & $.61(.22)$ & $.77(.22)$ & $.59(.24)$ & $.61(.23)$ \\
\hline \multicolumn{5}{|c|}{ Experiment 2: $20 \%$ of contrast difference } \\
\hline Preference & $.66(.18)$ & $.52(.21)$ & $.63(.26)$ & $.57(.25)$ \\
\hline Recognition & $.62(.25)$ & $.79(.18)$ & $.65(.26)$ & $.59(.27)$ \\
\hline \multicolumn{5}{|c|}{ Experiment 3: $40 \%$ of contrast difference } \\
\hline Preference & $.60(.20)$ & $.60(.18)$ & $.64(.25)$ & $.61(.24)$ \\
\hline Recognition & $.60(.26)$ & $.80(.15)$ & $.64(.22)$ & $.60(.34)$ \\
\hline
\end{tabular}

Notes. Standard deviations are in parentheses. 
pairs where the distractor was also made easier to process because it had a higher contrast than the target (Target-/Distractor + pairs, $t(13)=-0.191, p>.1)$.

\subsubsection{Recognition performance}

We observed significant recognition (subjects selected the targets in a proportion greater than chance), with medium-sized effects for Target-/Distractor- $(t(13)=1.807, p<.05, d=.48)$, and for $T$ Target $+/$ Distractor $+(t(13)=1.847, p<.05, d=.49)$, and a large effect for Target $+/$ Distractor $-(t(13)=4.618, p<.001, d=1.23)$. These three conditions were not significantly different $(p>.1)$. However, as with the mere exposure effect, we observed a particularly large recognition effect size for Target + /Distractor - pairs. Moreover, we observed no significant recognition for the Target-/Distractor + condition $(t(13)=1.370, p>.1, d=.36)$.

\subsubsection{Remember, Know, and Guess responses}

Table 2 presents the proportions of selected targets that were assigned Remember, Know, and Guess (RKG) responses for the four contrast conditions.

Separate ANOVAs with contrast condition as within-subject variable were performed for each response type. Regarding responses for targets (hits), there was a marginally significant contrast effect $(F(3,39)=2.773, p=.054)$ on $\mathrm{R}$ responses. This effect showed an advantage for Target + / Distractor - pairs in comparison to other pairs $(p \mathrm{~s}<.05)$. A similar contrast effect was found for $\mathrm{K}$ responses $(F(3,39)=4.624, p=.007)$, with more $\mathrm{K}$ responses for Target + /Distractor- pairs than for Target-/Distractor+ pairs $(p<.001)$. Jacoby, Yonelinas, and Jennings (1997) have suggested that, if recollection and familiarity are independent processes that operate in parallel, the contribution of familiarity to recognition is underestimated by the proportion of $\mathrm{K}$ (familiar) responses. When familiarity was measured by their Independence Remember/Know (IRK) procedure (Familiarity $=$ proportion of $\mathrm{K}$ responses $/ 1$ - proportion of $\mathrm{R}$ responses), the analyses revealed similar contrast effects $(F(3,39)=4.268, p=.01)$, with greater familiarity for Target + / Distractor - pairs than all other pairs (Target-/Distractor $+, F(3,39)=9.607, p=.008$; Target $+/$ Distractor $+, F(3,39)=3.482, p=.08$; target $-/$ Distractor $-, F(3,39)=6.566, p=.02)$. Thus, the greater recognition effect size for Target + /Distractor- pairs observed for recognition performance can be explained by both $\mathrm{R}$ responses (recollection-based recognition) and $\mathrm{K}$ responses (familiarity-based recognition).

Finally, there were no significant effects on $\mathrm{G}$ responses $(p>.05)$. Analyses did not reveal any significant effect of contrast on distractors that were falsely recognized and classified as $\mathrm{RKG}$ responses $(p s>.05)$.

Table 2

Proportions of Remember-Know-Guess responses for targets as a function of the four contrast conditions in Experiment 1

\begin{tabular}{lllll}
\hline & \multicolumn{2}{l}{ Distractors -} & & \multicolumn{2}{l}{ Distractors +} \\
\cline { 2 - 5 } & Targets - & Targets + & Targets - & Targets + \\
\hline Remember & $.25(.09)$ & $.35(.15)$ & $.29(.10)$ & $.22(.17)$ \\
Know & $.26(.12)$ & $.34(.10)$ & $.17(.13)$ & $.28(.11)$ \\
Guess & $.10(.10)$ & $.09(.09)$ & $.13(.10)$ & $.11(.13)$ \\
\hline
\end{tabular}

Notes. Standard deviations are in parentheses. 
In this first experiment, we observed a great explicit preference for pairs where the target was facilitated by both pre-exposure and high contrast. We observed a similar pattern of results for recognition, with the same pairs being perceived as more familiar. In addition, when the distractor was also made easy to process because it had a higher contrast than the target, the mere exposure effect disappeared and recognition performance declined.

Thus, an imperceptible manipulation of contrast at the test time seems to have an independent and direct impact on preference judgment and familiarity-based recognition that can be added to the pre-exposure effect. Consequently, when there is an imperceptible difference in contrast between stimuli, participants seem not to construct expectations concerning the processing ease of different classes of stimuli. In this context, people seem to base their judgment on a direct assessment of absolute fluency.

In our second experiment, we examined whether a more perceptible, but still slight, difference in the quality of stimuli during the preference judgment could produce an opposite effect. In other words, the question addressed in Experiment 2 was whether processing fluency due to pre-exposure was more likely to be used for judgments when picture quality would prompt participants to expect to be able to process targets less efficiently relative to distractors (Target-/Distractor + ) than when subjects construct larger expectations about target fluency (Target + (Distractor-).

\section{Experiment 2}

In Experiment 2, we examined cases in which a more perceptible figure-ground contrast variation at the time of test might create expectations about the processing of high- and low-quality stimuli. Contrast manipulation that favors the target, but not the distractor, may allow participants to formulate high expectations about target fluency. Thus the enhancement of fluency due to pre-exposure might go unnoticed and thus be less likely to be used for judgments. Conversely, with a high-contrast distractor, actual target fluency could be more important than the fluency normally expected when the target has a lower contrast than when it is as high contrast as the distractor. This possible discrepancy between expected fluency and actual fluency should result in an increase in positive judgment. So, while we observed a positive and direct impact of contrast advantage for targets in Experiment 1, we expected to find the opposite effect in the second experiment.

\subsection{Method}

\subsubsection{Subjects}

Twenty-four undergraduate students were volunteers in this experiment. There were 12 men and 12 women. Their mean age was 23.7 years $(S D=2.6)$.

\subsubsection{Materials and procedure}

The experimental details were the same as in Experiment 1, with one exception: low-contrast objects underwent a 20\% contrast (contrary to $10 \%$ in Experiment 1). These picture modifications were slight; thus, the decrease in contrast quality could be perceived without necessarily denoted. 


\subsection{Results and discussion}

The means as a function of the four contrast conditions are presented in Table 1 for preference and recognition. Again, as there was no effect of the order of the tasks on preference and recognition scores $(p s>.1)$, we did not consider this variable in the following analyses.

\subsubsection{Mere exposure effect}

We observed a significant and large mere exposure effect for Target-/Distractor- pairs $(t(23)=4.271, p<.001, d=.89)$. Contrary to Experiment 1 , we also found a medium effect for Target-/Distractor + pairs $(t(23)=2.303, p<.025, d=.48)$. For these Target-/Distractor + pairs, the fluency advantage of target over distractor was probably slighter than for other pairs. Nevertheless, if the enhanced fluency due to a prior repeated global encoding (i.e., processing facilitation through structural extraction induced by the orientation task; Schacter et al., 1990) is greater than the perceptual fluency due to the contrast level (i.e., processing facilitation through very low-level perceptual processes), then a previously seen low-contrast target should be perceived as slightly easier to process than a new high-contrast distractor. While this slight advantage of absolute fluency did not ensure to observe a mere exposure effect in Experiment 1, given the more perceptible quality difference in Experiment 2, this slight fluency advantage could be more surprising and thus more salient.

As expected, a visible high-contrast advantage for the target undermines the mere exposure effect (Target $+/$ Distractor - pairs: $t(23)=0.385, p>.1, d=.20)$. This result might suggest, on one hand, that subjects expected a greater target fluency advantage for these pairs and therefore might have attributed their entire actual fluency feeling to picture quality. On the other hand, contrast might be perceived as irrelevant in a preference judgment concerning a complex three-dimensional shape. In this context, high-processing fluency attributed to this irrelevant variable might be judged as uninformative and thus would not give rise to a feeling of preference. Consequently, subjects might turn to other inputs than fluency to make their judgments.

Finally, and more surprisingly, we observe an absence of effect for Target $+/$ Distractor + pairs $(t(23)=1.317, p>.1, d=.27)$. Indeed, in terms of absolute fluency, pre-exposure should induce a fluency advantage for the target over the distractor. In light of the attributional processes that might moderate the impact of fluency, subjects should not have formulated higher expectations concerning target fluency based on a comparison of the target and its distractor. However, given that contrast seems to be perceived as irrelevant to judgment, we assumed that subjects might have judged the fluency to be an uninformative cue for all high-contrast figures. In fact, participants may have based their fluency expectations on overall high- and low-quality pictures, rather than on a comparison between the target and distractor in each pair.

\subsubsection{Recognition performance}

Subjects selected the targets in a proportion greater than chance in each condition $(p s \leqslant .05)$, with medium-sized effects for Target-/Distractor- $(t(23)=2.352, p<.05, d=.49)$, and for Target-/Distractor $+(t(23)=2.826, p<.005, d=.59)$, a small effect for Target $+/$ Distractor $+(t(23)=1.763, p<.05, d=.37)$, and the large effect for Target $+/$ Distractor $-(t(23)=7.892$, $p<.001, d=1.64)$. An ANOVA revealed a significant effect for contrast $(F(3,69)=4.401$, $p=.006)$, with an advantage for Target $+/$ Distractor - pairs compared with other pairs $(p \mathrm{~s}<.05)$. 


\subsubsection{Remember, Know, and Guess responses}

Table 3 shows the proportions of selected targets that were assigned R, K, and $\mathrm{G}$ responses for the four contrast conditions.

Regarding the correct $\mathrm{R}$ responses, we observed a significant main effect for contrast $(F(3$, $69)=7.940, p<.001)$, indicating an advantage for the Target $+/$ Distractor - pairs compared with other pairs ( $p s \leqslant .001)$, as in Experiment 1 . This condition therefore seems to be an optimal condition for effective recollection-driven recognition. These pairs might constitute the easiest situation for recollection memory, because the target might completely match the memory trace (same object associated with same contrast context), whereas the distractor might not match it at all (different object with different context).

For correct $\mathrm{K}$ responses, we observed a significant main effect for contrast $(F(3,69)=5.794$, $p=.001)$ indicating an advantage for the Target-/Distractor + and Target-/Distractor- pairs compared with the other two kinds of pairs $(p s \leqslant .001)$. When familiarity was measured by the IRK procedure (Jacoby et al., 1997), the analyses confirmed these results. Consequently, contrary to Experiment 1, the better recognition performance for Target $+/$ Distractor - might be explained only by $\mathrm{R}$ responses (recollection-based recognition). These findings were thus similar to those for the mere exposure effect.

Finally, there were no significant effects on $\mathrm{G}$ responses $(p>.05)$. For distractors falsely recognized and classified as RKG responses; as in Experiment 1, analyses did not reveal any significant effect of contrast $(p \mathrm{~s}>.05)$.

Thus, we observed in Experiment 2 that a perceptible quality manipulation during the preference and recognition judgments has an inverse impact on preference as well as on familiaritybased recognition. This is consistent with the fluency-attribution account of the mere exposure effect and familiarity-based recognition described above, as well as the idea that the impact of experienced fluency on familiarity and explicit preference may be moderated by indirect factors (e.g., Bornstein \& D’Agostino, 1994; Butler et al., 2004; Reber et al., 2004; Schwarz, 2004a; Van den Bergh \& Vrana, 1998). The greater difference between stimuli seems to allow participants to construct expectations about the processing of high- and low-quality stimuli. In this context, people seem not to make their judgment based on a direct assessment of fluency per se, but rather on the fact that the fluent processing occurs under unexpected circumstances (see also Butler et al., 2004). However, these attributional processes seem to be implicit. Indeed, no participant was able to report on it and only three subjects pointed out the variation in contrast during the debriefing.

Moreover, since pre-exposure did not influence the preference judgment on high-quality stimuli, it seems that once a subject detected any variable that might produce fluency for the wrong

Table 3

Proportions of Remember-Know-Guess responses for targets as a function of the four contrast conditions in Experiment 2

\begin{tabular}{|c|c|c|c|c|}
\hline & \multicolumn{2}{|c|}{ Distractors - } & \multicolumn{2}{|c|}{ Distractors +} \\
\hline & Targets - & Targets + & Targets - & Targets + \\
\hline Remember & $.32(.21)$ & $.53(.23)$ & $.28(.23)$ & $.31(.21)$ \\
\hline Know & $.23(.19)$ & $.11(.14)$ & $.27(.24)$ & $.13(.13)$ \\
\hline Guess & $.08(.12)$ & $.16(.16)$ & $.10(.13)$ & $.16(.17)$ \\
\hline
\end{tabular}

Notes. Standard deviations are in parentheses. 
reason (i.e., high contrast), overall fluency was attributed to this variable. Thus, subjects might detect the influence of this variable and develop indefinite expectations from it but might not assess the exact extent of its impact. In a third experiment, the question addressed was whether participants become able to accurately estimate the effect of contrast on their feelings of fluency and to develop clear expectations based on this factor when a more obvious difference in contrast makes them really aware of a contrast that may result in fluency.

\section{Experiment 3}

In the third experiment, we directly tested the idea that a perceptible and obvious contrast difference at the test time could create expectation but with a more efficient estimation of the contrast effect. Indeed, subjects could more accurately assess the amount of fluency resulting from contrast by comparing the fluency for a specific stimulus to that of other stimuli with the same contrast level. In other words, the discrepancy between expected and actual fluency of processing may influence the preference judgment and the feeling of familiarity (as in Experiment 2) only when the variable that prompts subjects' expectations is subtle and tricky to assess. If this assumption is correct, then it should be possible to make preference and familiarity similar for high- and low-contrast stimuli by making the variation in contrast more obvious to subjects.

\subsection{Method}

\subsubsection{Subjects}

Fourteen young adults were volunteers in this experiment. There were seven men and seven women. Their mean age was 20.9 years $(S D=1.54)$.

\subsubsection{Materials and procedure}

The experimental details were the same as Experiment 1, with one exception: contrast variation was $40 \%$ (compared to $10 \%$ in Experiment 1 and to $20 \%$ in Experiment 2). This variation was marked and striking.

\subsection{Results and discussion}

The mean proportions of target objects selected in the recognition and preference tasks as a function of the four contrast conditions are presented in Table 1. As was the case in Experiments 1 and 2 since there was no effect of the order of the tasks on preference and recognition scores ( $p$ s $>.15$ ), we did not consider this variable in the following analyses.

\subsubsection{Mere exposure effect}

Subjects selected the targets in a proportion greater than chance for all pairs, with mediumsized effects (Target-/Distractor-, $t(13)=1.947, \quad p<.05, d=.54 ; \quad$ Target $+/$ Distractor - , $t(13)=2.133, p<.05, d=.59$; Target-/Distractor $+, t(13)=2.135, p<.05, d=.59$; Target $+/$ Distractor $+, t(13)=1.771, p=.05, d=.49)$. Thus, we observed a significant mere exposure effect for each condition. Moreover, an ANOVA with contrast condition as within-subject variable did 
Table 4

Proportions of Remember-Know-Guess responses for targets as a function of the four contrast conditions in Experiment 3

\begin{tabular}{llllll}
\hline & \multicolumn{2}{l}{ Distractors -} & & \multicolumn{2}{l}{ Distractors +} \\
\cline { 2 - 3 } & Targets - & Targets + & & Targets - & Targets + \\
\hline Remember & $.34(.24)$ & $.49(.20)$ & $.19(.18)$ & $.31(.23)$ & $.30(.24)$ \\
Know & $.19(.18)$ & $.11(.13)$ & $.17(.17)$ & $.20(.19)$ \\
Guess & $.06(.09)$ & & & $.11(.13)$ & $.10(.10)$ \\
\hline
\end{tabular}

Notes. Standard deviations are in parentheses.

not reveal significant effect $(F(3,39)=0.186, p=.91)$. So the effect of contrast variation on the preference judgment disappeared when the contrast manipulation was striking.

\subsubsection{Recognition performance}

Subjects selected the targets in a proportion greater than chance in each condition $(p s \leqslant .05)$. An ANOVA revealed a marginally significant contrast effect $(F(3,39)=2.492, p=.07)$. This effect indicated a slight advantage for Target $+/$ Distractor - pairs compared with other pairs $(p \mathrm{~s}<.07)$.

\subsubsection{Remember, Know, and Guess}

Regarding the correct $\mathrm{R}$ responses (see Table 4), we observed a marginally significant effect of contrast $(F(1,13)=2.470, p=.076)$, with Target $+/$ Distractor - pairs slightly superior to other pairs $(p s \leqslant .07)$.

No significant effect was observed for correct $\mathrm{K}$ and $\mathrm{G}$ responses $(p \mathrm{~s}>.1)$. The absence of any effect of contrast on $\mathrm{K}$ was also observed with the IRK procedure $(p=.27)$. Thus, the contrast effect on familiarity disappeared, just as it did with the preference judgment.

During the debriefing following the experiment, subjects noted that the darkest figures were less easy to process and that the preference judgment about Target $+/$ Distractor - or Target-/Distractor + pairs was more difficult than with Target-/Distractor - and Target $+/$ Distractor + pairs. In other words, when subjects become really aware of contrasts that may produce fluency, they seem to have more motivation and also to allocate more resources to accurately estimate the size of the contrast effect. These effects highlight the importance of the "relativity" of fluency. In fact, the feeling of fluency is experienced in comparison to the relative difficulty of the processing of other items. It seems that awareness of the exact influence of contrast depends on the overall comparison of stimuli within the same class. This comparison may allow participants to make accurate forecasts concerning the processing fluency norm for each type of stimuli. Awareness of the exact contrast influence thus seems to ensure that participants will allocate the portion of fluency that is not explained by contrast (i.e., in fact, due to pre-exposure) to preference or familiarity.

\section{Conclusion}

In these experiments, the influence of processing fluency due to pre-exposure on preference and recognition judgments was found to depend on the quality of the target and distractor items 
presented in the test phase (manipulated by means of variation in figure-ground contrast). Nonetheless, the influence of stimulus quality on the prior exposure effect was expressed in different ways depending on the subjects' awareness of the influence of this variable on their own processing experience. In Experiment 1, the influence of an imperceptible difference in stimulus quality was direct. So, for a portion of the target-distractor pairs, the contrast advantage for targets might make the fluency feeling particularly strong, greater on average than for matched distractors or for other stimuli in the test phase. In that case, it is the speed and ease of processing resulting from both repeated exposure (see Jacoby \& Dallas, 1981) and high-quality stimuli (e.g., Checkosky \& Whitlock, 1973; Whittlesea et al., 1990) that influence people's decision processes: the absolute magnitude of fluency resulting from the various sources can have a direct impact on people's preference and recognition decisions. These findings tally, on the one hand, with the fluency account of the mere exposure effect (Bornstein \& D'Agostino, 1994; Jacoby et al., 1989; Seamon et al., 1983a, 1983b; Whittlesea, 1993; Whittlesea \& Price, 2001), and on the other hand, with the well-established idea that fluency may serve as a cue for various judgments (such as recognition, through the familiarity feeling, e.g., Jacoby \& Dallas, 1981; Whittlesea et al., 1990).

In Experiment 2, with a more perceptible but still unnoticed quality difference at the time of testing, the pre-exposure influence was particularly great when target stimuli were presented with a low-quality level. In that case, it is the surprise related to the discrepancy between what is expected of the type of stimulus and what actually happens in processing that sponsors the attribution process moderating the role of fluency in preference and recognition judgment (Whittlesea \& Williams, 1998, 2000, 2001a, 2001b). In other words, this experiment shows that people are able to use the surprising fluency when the quality of an event cannot be reconciled with the actual feeling of fluency (see Whittlesea \& Leboe, 2003). Conversely, when the difference in picture quality was really marked (Experiment 3), a similar pre-exposure effect was noted for both high- and low-quality stimuli. We therefore speculated that the expectation effect in Experiment 2 occurred because subjects developed an indefinite expectation about the amount of fluency related to the two classes of stimuli.

In sum, these experiments suggested that when people are not able to distinguish between the various sources of fluency (e.g., indistinct quality picture), they can simply base their judgment on the experienced fluency amount. When people are able to distinguish a fluency source (e.g., highquality level) and build up their expectations about how the different classes of stimuli should be processed, then the use of fluency for their judgment may depend on the accuracy of their expectations. With a subtle fluency source, people may develop indefinite expectations and attribute their feeling of fluency entirely to this source. In this case, they may turn to other cues than this expected fluency to make their judgments. With an obvious fluency source, people may become aware of the specific fluency normally associated with the two stimuli types. In this case, they may be perfectly able to detect fluency that is not explained by this source and will attribute this portion to familiarity or to the desirable nature of the stimulus.

This study, along with the results of several other recent studies (e.g., Butler et al., 2004; Whittlesea \& Leboe, 2003; Whittlesea \& Williams, 1998, 2000, 2001a, 2001b; see also Westerman et al., 2002), supports the notion that familiarity and preference are a function of the perceiver's dynamic attributional processes. That is, a stimulus may be perceived either as familiar and/or liked or as unfamiliar and/or unliked depending on the expectations and interpretations of the perceiver (Lloyd et al., 2003). The role of these attributional processes in the fluency-affect link has, at 
present, received no attention. Future work should therefore assess more directly the contribution of participants' expectations in the mere exposure effect. In line with Winkielman \& Fazendeiro's work (unpublished, cited by Reber et al., 2004) that demonstrates that the influence of fluency on liking judgments is eliminated when subjects attribute their first affective reaction to an irrelevant variable, instructional manipulation might conceivably address this issue.

Finally, theorists of aesthetics have long considered figure-ground contrast to be an "objective" determinant of beauty (see Reber et al., 2004). However, this study also supports the notion that the "contrast as liking" factor resides in the subjective eye of the perceiver. In addition, Reber and Schwarz (2001) showed that it is not the objective figure-contrast per se that is an attractive attribute, rather it is the resulting perceptual fluency which contributes to more positive evaluations. Our findings confirm this assumption. Moreover, while contrast seems to be judged pertinent by subjects for their preference judgments concerning relatively simple figures (Reber et al., 1998; Reber \& Schwarz, 2001), it seems to be perceived non-essential and incidental for complex figures that are richer in visual features (three-dimensional objects). Further, this study shows that, depending on their awareness of the influence of contrast on fluency, perceivers will attribute the fluency due to contrast to either the familiarity or the desirable attributes of a stimulus (resulting in more positive judgments), or to their own processing experience related to this irrelevant source (undermining or reducing the influence of fluency on judgments).

\section{Appendix A. Remember-Know-Guess instructions}

\section{A.1. Remember}

Often, when you recognize a figure, you remember that you have seen it before and you remember the precise context you have seen in it. You may also remember an event, a feeling or a thought that occurred when you first saw this figure. For example, when you saw that figure for the first time, you found that it looked like building, spaceship, etc. or you noticed a particular feature. So we ask you to classify as "remember" a response for which you can retrieve information about the encoding context.

\section{A.2. Know}

We ask you to classify a response as "know" if you do not remember any information associated with the figure. You are sure that you have seen it before because you have a strong feeling of familiarity, but you do not remember any information encoded with the figure, such as when you saw it or any element that you might have noticed.

\section{A.3. Guess}

You can guess if you do not remember any of the figures. If none of the figures seem familiar or if you do not remember any information about the learning context for any of them, you cannot respond that you "remember" or "know." Because you still have to choose one figure, you can guess. 


\section{References}

Berlyne, D. E., \& Crozier, J. B. (1971). Effects of complexity and prechoice stimulation on exploratory choice. Perception and Psychophysics, 10, 242-246.

Bornstein, R. F. (1989). Exposure and affect: Overview and meta-analysis of research, 1968-1987. Psychological Bulletin, 106, 265-289.

Bornstein, F. R., \& D’Agostino, P. R. (1992). Stimuli recognition and the mere exposure effect. Journal of Personality and Social Psychology, 4, 545-552.

Bornstein, R. F., \& D'Agostino, P. R. (1994). The attribution and discounting of perceptual fluency: Preliminary tests of a perceptual fluency/attributional model of the mere exposure effect. Social Cognition, 12, 103-128.

Bornstein, R. F., Kale, A. R., \& Cornell, K. R. (1990). Boredom as a limiting condition on the mere exposure effect. Journal of Personality and Social Psychology, 58, 791-800.

Butler, L. T., Berry, D., \& Helman, S. (2004). Dissociating mere exposure and repetition priming as a function of word type. Memory and Cognition, 5, 759-767.

Cacioppo, J. T., Petty, R. E., Losch, M. E., \& Kim, H. S. (1986). Electromyographic activity over facial muscle regions can differentiate the valence and intensity of affective reactions. Journal of Personality and Social Psychology, 50, 260-268.

Carrasco, M., \& Seamon, J. G. (1996). Priming impossible figures in the object decision test: The critical importance of perceived stimulus complexity. Psychonomic Bulletin and Review, 3, 344-351.

Checkosky, S. F., \& Whitlock, D. (1973). Effects of pattern goodness on recognition time in a memory search task. Journal of Experimental Psychology, 100, 341-348.

Cohen, J. (1988). Statistical power analysis for the behavioral sciences (2nd ed.). Hillsdale, NJ: Lawrence Earlbaum Associates.

Cox, D. S., \& Cox, A. D. (1988). What does familiarity breed? Complexity as a moderator of repetition effects in advertisement evaluation. Journal of Consumer Research, 15, 111-116.

Cox, D. S., \& Cox, A. D. (2002). Beyond first impressions: The effects of repeated exposure on consumer liking of visually complex and simple product designs. Journal of the Academy of Marketing Science, 30, 119-130.

Fenske, M. J., Raymond, J. E., \& Kunar, M. A. (2004). The affective consequences of visual attention in preview search. Psychonomic Bulletin and Review, 11, 1055-1061.

Gardiner, J. M. (1988). Functional aspects of recollective experience. Memory and Cognition, 16, 309-313.

Harmon Jones, E., \& Allen, J. J. B. (2001). The role of affect in the mere exposure effect: Evidence from psychophysiological and individual differences approaches. Personality and Social Psychology Bulletin, 27, 889-898.

Harrison, A. A. (1977). Mere exposure. In L. Berkowitz (Ed.). Advances in experimental social psychology (Vol. 10, pp. 39-83). New York: Academic Press.

Jacoby, L. R., \& Dallas, M. (1981). On the relationship between autobiographical memory and perceptual learning. Journal of Experimental Psychology: General, 3, 306-340.

Jacoby, L. L., Kelley, C. M., \& Dywan, J. (1989). Memory attributions. In F. I. M. Craik \& H. L. Roediger (Eds.), Varieties of memory and consciousness: Essays in honour of Endel Tulving (pp. 391-422). Hillsdale, NJ: Lawrence Erlbaum Associates.

Jacoby, L. L., \& Whitehouse, K. (1989). An illusion of memory: False recognition influenced by unconscious perception. Journal of Experimental Psychology: General, 118, 126-135.

Jacoby, L. L., Yonelinas, A. P., \& Jennings, J. M. (1997). The relation between conscious and unconscious (automatic) influences: A declaration of independence. In J. W. Schooler \& J. D. Cohen (Eds.), Scientific approaches to consciousness (pp. 13-47). Hillsdale, NJ: Lawrence Erlbaum Associates.

Kelley, C. M., \& Jacoby, L. L. (1998). Subjective reports and process dissociation: Fluency, knowing, and feeling. Acta Psychologica, 98, 127-140.

Kelley, C. M., \& Rhodes, M. G. (2002). Making sense and nonsense of experience: Attributions in memory and judgment. In B. H. Ross (Ed.). The psychology of learning and motivation: Advances in research and theory (Vol. 41, pp. 293-320). San Diego, CA, USA: Academic Press.

Lloyd, M. E., Westerman, D. L., \& Miller, J. K. (2003). The fluency heuristic in recognition memory: The effect of repetition. Journal of Memory and Language, 48, 603-614. 
Rajaram, S. (1993). Remembering and knowing: Two means of access to the personal past. Memory and Cognition, 21, $89-102$.

Rajaram, S., \& Geraci, L. (2000). Conceptual fluency selectively influences knowing. Journal of Experimental Psychology: Learning, Memory, and Cognition, 26, 1070-1074.

Reber, R., \& Schwarz, N. (2001). The hot fringes of consciousness: Perceptual fluency and affect. Consciousness and Emotion, 2, 223-231.

Reber, R., Schwarz, N., \& Winkielman, P. (2004). Processing fluency and aesthetic pleasure: Is beauty in the perceiver's processing experience? Personality and Social Psychological Review, 8, 364-382.

Reber, R., Winkielman, P., \& Schwarz, N. (1998). Effects of perceptual fluency on affective judgments. Psychological Science, 9, 45-48.

Schacter, D. L., Cooper, L. A., \& Delaney, S. M. (1990). Implicit memory for unfamiliar objects depends on access to structural descriptions. Journal of Experimental Psychology: General, 119, 5-24.

Schwarz, N. (2004a). Metacognitive experiences in consumer judgment and decision making. Journal of Consumer Psychology, 14, 332-348.

Schwarz, N. (2004b). Metacognitive experiences: Response to commentaries. Journal of Consumer Psychology, 14, 370-373.

Seamon, J. G., Brody, N., \& Kauff, D. M. (1983a). Affective discrimination of stimuli that are not recognized: Effects of shadowing, masking, and cerebral laterality. Journal of Experimental Psychology: Learning, Memory, and Cognition, 9, 544-555.

Seamon, J. G., Brody, N., \& Kauff, D. M. (1983b). Affective discrimination of stimuli that are not recognized II: Effects of delay between study and test. Bulletin of the Psychonomic Society, 21, 187-189.

Seamon, J. G., Ganor Stern, D., Crowley, M. J., Wilson, S. M., Weber, W. J., O’Rourke, C. M., et al. (1997). A mere exposure effect for transformed three-dimensional objects: Effects of reflection, size, or color changes on affect and recognition. Memory and Cognition, 25, 367-374.

Seamon, J. G., Williams, P. C., Crowley, M. J., Kim, I. J., Langer, S. A., Orne, P. J., et al. (1995). The mere exposure effect is based on implicit memory: Effect of stimulus type, encoding conditions, and number of exposures on recognition and affect judgments. Journal of Experimental Psychology: Learning, Memory, and Cognition, 21, $711-721$.

Tulving, E. (1985). Memory and consciousness. Canadian Psychology, 26, 1-12.

Van den Bergh, O., \& Vrana, S. R. (1998). Repetition and boredom in a perceptual fluency/attributional model of affective judgments. Cognition and Emotion, 12, 533-553.

Westerman, D. L. (2001). The role of familiarity in item recognition, associative recognition, and plurality recognition on self-paced and speeded tests. Journal of Experimental Psychology: Learning, Memory, and Cognition, 27, 723-732.

Westerman, D. L., Lloyd, M. E., \& Miller, J. K. (2002). The attribution of perceptual fluency in recognition memory: The role of expectation. Journal of Memory and Language, 47, 607-617.

Whittlesea, B. W. A. (1993). Illusions of familiarity. Journal of Experimental Psychology: Learning, Memory, and Cognition, 19, 1235-1253.

Whittlesea, B. W. A. (2003). On the construction of behavior and subjective experience: The production and evaluation of performance. In C. J. Marsolek \& J. S. Bowers (Eds.), Rethinking implicit memory (pp. 239-260). London: Oxford University Press.

Whittlesea, B. W., Jacoby, L. L., \& Girard, K. (1990). Illusions of immediate memory: Evidence of an attributional basis for feelings of familiarity and perceptual quality. Journal of Memory and Language, 29, 716-732.

Whittlesea, B. W. A., \& Leboe, J. P. (2003). Two fluency heuristics (and how to tell them apart). Journal of Memory and Language, 49, 62-79.

Whittlesea, B. W., \& Price, J. R. (2001). Implicit/explicit memory versus analytic/nonanalytic processing: Rethinking the mere exposure effect. Memory and Cognition, 29, 234-246.

Whittlesea, B. W. A., \& Williams, L. D. (1998). Why do strangers feel familiar, but friends don't? A discrepancyattribution account of feelings of familiarity. Acta Psychologica, 98, 141-165.

Whittlesea, B. W. A., \& Williams, L. D. (2000). The source of feelings of familiarity: The discrepancy-attribution hypothesis. Journal of Experimental Psychology: Learning, Memory, and Cognition, 26, 547-565. 
Whittlesea, B. W. A., \& Williams, L. D. (2001a). The discrepancy-attribution hypothesis: I. The heuristic basis of feelings and familiarity. Journal of Experimental Psychology: Learning, Memory, and Cognition, 27, 3-13.

Whittlesea, B. W. A., \& Williams, L. D. (2001b). The discrepancy-attribution hypothesis: II. Expectation, uncertainty, surprise, and feelings of familiarity. Journal of Experimental Psychology: Learning, Memory, and Cognition, 27, $14-33$.

Williams, P., \& Tarr, M. J. (1997). Structural processing and implicit memory for possible and impossible figures. Journal of Experimental Psychology: Learning, Memory, and Cognition, 23, 1344-1361.

Winkielman, P., \& Cacioppo, J. T. (2001). Mind at ease puts a smile on the face: Psychophysiological evidence that processing facilitation elicits positive affect. Journal of Personality and Social Psychology, 81, 989-1000.

Winkielman, P., \& Fazendeiro, T. A. (2003). The role of conceptual fluency in preference and memory. Unpublished manuscript.

Winkielman, P., Schwarz, N., Fazendeiro, T. A., \& Reber, R. (2003). The hedonic marking of processing fluency: Implications for evaluative judgment. In K. C. Klauer \& J. Musch (Eds.), The psychology of evaluation: Affective processes in cognition and emotion (pp. 189-217). Mahwah, NJ: Lawrence Erlbaum Associates.

Zajonc, R. B. (1968). Attitudinal effects of mere exposure. Journal of Personality and Social Psychology, Monograph Supplement, 9, 1-27. 